SUCCESS IN REFERENTIAL COMMUNICATION 


\section{PHILOSOPHICAL STUDIES SERIES}

\section{VOLUME 80}

Founded by Wilfrid S. Sellars and Keith Lehrer

Editor

Keith Lehrer, University of Arizona, Tucson

Associate Editor

Stewart Cohen, Arizona State University, Tempe

Board of Consulting Editors

Lynne Rudder Baker, University of Massachusetts at Amherst

Radu Bogdan, Tulane University, New Orleans

Allan Gibbard, University of Michigan

Denise Meyerson, University of Cape Town

Franois Recanati, École Polytechnique, Paris

Stuart Silvers, Clemson University

Nicholas D. Smith, Michigan State University

The titles published in this series are listed at the end of this volume. 


\section{SUCCESS IN REFERENTIAL COMMUNICATION}

by

MATTHIAS PAUL

Westfälische Wilhelms-Universität,

Münster, Germany

SPRINGER-SCIENCE+BUSINESS MEDIA, B.V. 
A C.I.P. Catalogue record for this book is available from the Library of Congress.

ISBN 978-90-481-5322-0 ISBN 978-94-017-3181-2 (eBook)

DOI 10.1007/978-94-017-3181-2

Printed on acid-free paper

\author{
All Rights Reserved \\ (C)1999 Springer Science+Business Media Dordrecht \\ Originally published by Kluwer Academic Publishers in 1999
}

No part of the material protected by this copyright notice may be reproduced or utilized in any form or by any means, electronic or mechanical, including photocopying, recording or by any information storage and retrieval system, without written permission from the copyright owner 
For Silja 


\section{CONTENTS}

Acknowledgements $\quad$ ix

Conventions $\quad$ xi

INTRODUCTION xiii

1. The Issue xiii

2. The Plan xviii

3. The Methodology xxii

4. The Empirical Relevance xxiv

CHAPTER 1 CHARACTERIZING REFERENTIAL COMMUNICATON 1

1. An Intuitive Characterization of Referential Communication 2

2. Reference to Particulars

3. Referring Expressions 19

CHAPTER 2 MENTAL STATES IN REFERENTIAL COMMUNICATION 31

1. A Representational Theory of Mental States 31

2. The Synchronic Features of Ideas $\quad 37$

3. The Diachronic Features of Ideas and Donnellan's Distinction 40

CHAPTER 3 RE-IDENTIFICATION IN REFERENTIAL 49 COMMUNICATION

1. Models of Re-Identification in Referential Communication 50

2. Is Re-Identification Required for Communicative Success? 55

3. Does it Matter How Re-Identification Gets Established? 60

CHAPTER 4 ACCOUNTING FOR MENTAL REFERENCE 68

1. Satisfactional Accounts of Mental Reference $\quad 69$

$\begin{array}{ll}\text { 2. Causal Accounts of Mental Reference } & 73\end{array}$

3. Hybrid Accounts of Mental Reference $\quad 80$

CHAPTER 5 TRADITIONAL ACCOUNTS OF SUCCESS IN 89 REFERENTIAL COMMUNICATION

1. Object-Dependent Accounts of Communicative Success 90

1.1 The Problem of Communicative Failure 91

Despite Sameness in Reference

1.2 The Problem of Communicative Success without 95

Reference

1.3 A Meinongean Defense 97 
viii

\section{CONTENTS}

2. Fregean Accounts of Communicative Success $\quad 100$

2.1 The Problem of Diversity 102

2.2 The Similarity Strategy and Why it Fails 108

CHAPTER 6 EVANS' ACCOUNT OF SUCCESS IN REFERENTIAL 113 COMMUNICATION

1. Genuine and Quasi Referential Communication 114

2. The Argument from Understanding $\quad 120$

3. Evan's Argument from Unity 129

CHAPTER 7 A NEW ACCOUNT OF SUCCESS IN REFERENTIAL 136 COMMUNICATION

1. External Success in Referential Communication 138

$\begin{array}{ll}1.1 \text { Different Kinds of Referential Communication } & 138\end{array}$

1.2 Agents Relating to Practices of Object-Pretence 146

2. Entertaining the Right Modes of Presentation 152

in Referential Communication

2.1 Evans' and Recanati's Success Conditions 154

2.2 An Action-Based Success Condition $\quad 158$

3. Summary

163

$\begin{array}{ll}\text { References } & 165\end{array}$

$\begin{array}{ll}\text { Index } & 169\end{array}$ 


\section{ACKNOWLEDGEMENTS}

This book has come into being over a long time and at different places. The background for it was laid in Bielefeld, where I studied philosophy from 1985-1991. The topic came up in conversations with John Perry during my time as an exchange student at Stanford University in 1987/88. Yet I did not start to work on the topic before 1992 when I began a PhD project at the Centre of Cognitive Science in Edinburgh, which led to my dissertation in 1996. The book is firmly based on the dissertation, which explains the coincidence in titles, though they are not identical. Many gaps have been filled in and some erroneous material has been taken out during the last two years at the philosophy department of the University of Münster. Thanks are due to all those who helped with advice, comments and encouragement at the various stages of the project. To start with, I am grateful to Robin Cooper and Paul Schweizer, my principal supervisors at the Centre for Cognitive Science. Both commented on earlier chapters and draft versions of the dissertation. Thanks are also due to the examiners Andrew Woodfield and Jon Oberlander for making me re-work certain parts of the dissertation. Further I want to thank Kent Bach, Marcus Birke, Christopher Gauker, Ruth Millikan, Francois Recanati, Rosemarie Rheinwald and Marcus Willaschek who all made helpful suggestions in the process of turning the dissertation into a book. I am also grateful to Peter Ruhrberg and Silja Vöneky for discussing with me the same example cases, and the different solutions I came up with, over and over again. Finally, I want to thank Alistair Knott for proofreading various draft versions of the book. 


\section{CONVENTIONS}

For clarity and simplicity I have adopted the following conventions: italics are used for variables and to highlight important notions, in particular when they get introduced. Single quotes are put around words or sentences that are being talked about. Double quotes are used for direct quotation, and to register a deliberate distance from a particular phrasing. The pronoun 'he' is normally used without the implication of gender. 


\section{INTRODUCTION}

Of all affairs, communication is the most wonderful. That things should be able to pass from the plane of external pushing and pulling to that of revealing themselves to man, and thereby to themselves; and that the fruit of communication should be participation, sharing, is a wonder by the side of which transubstantiation pales.

John Dewey (1925)

\section{THE ISSUE}

People communicate with each other by using language, for instance by making statements like (1) or asking questions like (2):

(1) Caesar was murdered.

(2) Who murdered Caesar?

In communicating with each other we can succeed, and in fact seem to do so most of the time, but we can also fail. Commonly it is said that for communication to succeed, the hearer must understand the speaker's words. This raises the question, under which conditions a hearer can be credited with understanding the speaker's words. For instance, what must be the case in order that someone can be credited with understanding my assertion of (1).

In this work the question regarding what understanding in communication consists in will be addressed in more detail. Yet it will not be addressed in its most general form, but with regard to a certain type of communication, namely referential communication. By this I mean those communicative acts where a speaker uses a singular term or another expression with the intention to refer an audience to a particular object he, the speaker, has in mind. For instance in uttering (1) or (2) I am using the proper name 'Caesar' in such a referring or referentially intended way. Now whether one understands these utterances depends in part on the success of the "constituent" referring acts. That is, without understanding my use of the proper name 'Caesar' one will not understand my assertions of (1) or (2), at 
least not in any complete sense. ${ }^{1}$ The central question that I will address in this work concerns the conditions under which a referring act or simply referential communication is successful. That is, what must be the case for a hearer to be credited with understanding the referential use of a singular term by a speaker?

Prima facie one might think that answering this question should be quite easy. For given that the speaker's underlying aim in a referring act is to refer a hearer to a particular object, then the hearer will have understood such an act if and only if he recognizes or identifies the respective object the speaker intended him to refer to. That is, he must simply come to entertain a thought or come to be in some mental state which refers to the same object as does the thought or mental state which underlies the speaker's referring act. For instance you will have understood my referential use of 'Caesar' in asserting 'Caesar got murdered' if and only if you come to entertain a thought which refers to the same object as does my underlying thought, which is the Roman emperor Caesar.

Despite its intuitive plausibility this simple object-dependent account of success in referential communication - which makes communicative success dependent on reference to particular objects in the world - faces severe problems. One central problem arises with regard to Frege-style examples of the following kind: imagine that you come across one of those shops which have a TV screen in the window connected to a camera that shows all the people passing by. In this particular case the camera is installed in such a way that you can only see your back on the screen, and thus it can happen quite easily that although you see yourself you do not recognize the person you see as yourself. Now assume that on a crowded shopping day you are just passing by that window and you hear a man saying near to you:

\section{(3) You have lost your bag!}

Now it is possible that you see the man who said this on the TV screen and you also see the person who is being addressed, but you do not recognize that it is you who is being addressed. In such a case you will come to entertain a thought which refers to the same object as does the speaker's thought, namely to you, yet in a crucial sense you do not understand his referential use of the pronoun 'You'. For although you perceptually recognize the person being addressed you do not recognize that it is you who is being addressed. Yet it seems that just this would be required for complete

\footnotetext{
' Throughout this work the terms 'referring' act and 'referential communication' will be used interchangeably.
} 
communicative success. This shows that requiring of the hearer to entertain a thought which refers to the same object as the speaker's thought cannot be sufficient for the hearer's understanding of the speaker's referring act. ${ }^{2}$

In the light of such Frege-style examples some have proposed to modify the above object-dependent account by making communicative success also dependent on the entertained modes of presentation under which the communicating agents think of the purported referent. Such a Fregean move has been made for instance by Evans (1982) who writes:

"... understanding the remarks we are concerned with requires not just that the hearer think of the referent, but that he think of it in the right way." (p. 315)

Thus in order to be credited with understanding a referring act, the hearer must, at least in some cases, not only entertain a thought that refers to the same object as does the speaker's underlying thought, but in addition the referent must also be presented to him in a certain way; that is, he must think of it under a certain mode of presentation. For instance in the above cameraexample communication fails because the hearer does not come to think of the referent in the right way, which would be something like the "self-mode of presentation", whatever that is. Thus according to such a proposal, which has also been put forward by Recanati (1993) recently, entertaining thoughts with the same referent will only be a necessary criterion for successfully understanding a referring act, but not a sufficient one.

Unfortunately, even this modified object-dependent account of understanding referring acts faces severe problems. First of all, it is not clear what the relevant modes of presentation are which are required for the understanding of the various kinds of singular terms. But more importantly, as certain examples suggest, agents can understand referring acts even if they are entertaining thoughts which do not refer to any objects in the world at all, and thus sameness in reference could not even be a necessary condition for communicative success. Imagine for instance that there had never been an ancient Greek city called 'Troy' and that all the events that allegedly had taken place in connection with this city had entirely been invented by Homer. This would have the consequence that in using the name 'Troy' as in (4) we would up to now always have failed to refer to any particular place or object:

(4) Troy was discovered by Schliemann.

\footnotetext{
${ }^{2}$ This example has been inspired by the well known examples involving indexicals by Perry (1979).
} 
Nevertheless it seems that we have perfectly understood each other when using this name in communication. For whether the name 'Troy' has a referent seems in a certain sense irrelevant for the success of our communicative efforts. Thus, what this and similar examples suggest is that coming to entertain thoughts which refer to the same object in the world cannot even be a necessary condition for the understanding of referentially used singular terms, as assumed above under the modified object-dependent account.

What might be suggested in light of these problems is to account for success in referring acts solely in terms of Fregean modes of presentation under which agents are assumed to think of the alleged referents of singular terms. For it seems plausible that agents can attach them to referential uses of such expressions even when their underlying thoughts refer to no objects in the world. For instance our uses of the name 'Troy', even if they had been empty, would nevertheless have involved certain modes of presentation of a city. Accordingly it seems promising to account for understanding referring acts solely in terms of such modes of presentation. This kind of proposal has been associated most prominently with the work of Frege from which the following account can be derived: a hearer will have understood the referential use of a singular term $t$ by a speaker if he comes to attach the same mode of presentation to $t$ that was attached to it by the speaker. ${ }^{3}$ If we assume, as many have done, that these modes of presentation can at least in part be given descriptively it follows that a referring act can only succeed if speaker and hearer are attaching the same descriptions or predicates to the speaker's referring expression, or more precisely descriptions or predicates with the same content. ${ }^{4}$ For instance with the proper name 'Troy' different agents might associate the descriptions 'being a city', 'being located in ancient Greece', 'being the place where the Troyan war took place', 'being described by Homer'. In principle this success condition allows us to account for those empty cases in which the communicating underlying thoughts do not refer to the objects they allegedly refer to, for instance to what is called by 'Troy'. For agents might attach the same descriptions to a used singular term and thus, according to such a Fregean account of understanding referring acts, their communicative efforts succeed although their underlying thoughts do not refer to any objects in the world.

\footnotetext{
${ }^{3}$ See for instance Dummett (1981), Evans (1982) and McCulloch (1995) for more general statements of what they have called the Fregean account or theory of communication or understanding.

${ }^{4}$ For the moment the idealizing assumption will be made that different agents always attach the same content to a description or predicate they use, for instance to the predicates 'being male', 'being Greek', etc.
} 
Unfortunately, this Fregean account also faces a central problem, namely it seems far too strong as an account of communicative success in referring acts. For according to it, speaker and hearer always had to entertain the same modes of presentation for communication to succeed yet in many cases we seem to be able to achieve success although we think of the alleged referent in quite different ways. For instance you might think of Prince Charles as the prince of Wales who studied at Cambridge and who committed adultery whereas I simply think of him as the son of Queen Elisabeth II. Then according to the Fregean account, referential communication between us involving the name 'Prince Charles' could not be successful, which seems highly counterintuitive.

Having reached this stage of the discussion we seem to be faced with the following dilemma: although human subjects are obviously able to understand referential uses of singular terms in communication, it is not clear at all what their understanding might consist in. For, as the discussion has shown, several intuitively plausible and also prominent accounts seem to fail in this regard. They simply do not match certain intuitions we have regarding agents succeeding or failing in referential communication. These intuitions can be summarized as follows:

Frege-Intuition: A hearer can fail to understand the referential use of a singular term by a speaker although they are entertaining thoughts that refer to the same object in the world (camera-example).

Empty-Case-Intuition: A hearer can understand the referential use of a singular term by a speaker although they are entertaining thoughts that refer to no object in the world (troy example).

Diversity-Intuition: A hearer can understand the referential use of a singular term by a speaker although they are not attaching the same modes of presentation to it (Prince Charles example).

The question is whether any of the accounts outlined above can somehow be brought into harmony with these intuitions or whether they all fail in this regard and a new account is needed. In later chapters these accounts will be discussed in more detail and possible lines of defense will be considered. The following section will give a more detailed overview on how the question of understanding referring acts will be tackled in this work. 


\section{THE PLAN}

In chapter $l$ the attempt will be made of making the phenomenon of referring acts, or of referential communication, more precise. I will start out with the intuitive characterization of referring acts in terms of referential intentions to refer an audience to particular things one has in mind. Although this characterization will allow us to draw certain important distinctions, as it stands it is quite imprecise since it rests on the vague notion of "having a thing in mind". The question is whether or not some more explanatory characterization of referring acts can be given. In this regard the role of two important notions will be explored, first that of reference and then that of a referring expression. For it seems not implausible that referring acts always involve reference to some objects, and further that they are necessarily performed by referring expressions. Yet what the discussion will show is that neither of these assumptions holds true. Agents can perform referring acts without actually referring to some objects, at least given the standard conception of "an object of reference". And secondly, they can perform referring acts without actually using what has commonly been considered as a referring expression.

In chapter 2 it will be argued that referring acts are best characterized in terms of the underlying mental states of the communicating agents. More precisely, it will be argued that there are certain representations for particular objects, called ideas, which are distinctive of the mental states that underlie referring acts. That is, in performing a referring act a speaker is entertaining a certain idea which he aims to "reproduce" in some way in the hearer, and in understanding the referring act the hearer must come to entertain an idea as well, namely one which relates in a certain way to the speaker's idea. The main purpose of chapter 2 is to make the nature of these ideas and the proposed characterization of referring acts in terms of them more precise. In this regard I will first point out what I take to be their central synchronic features, in particular explore the widely held conception of ideas as being associated with some sort of object-files. Further, their diachronic features will be discussed in some detail. In this context a distinction between two kinds of ideas will be proposed which will throw some new light on Donnellan's referential/attributive distinction.

It seems plausible that in many cases of referential communication the hearer will not come to entertain a new idea, but he will link the speaker's utterance to an idea which he already possesses. That is, he will re-identify in some way the object the speaker intends to relate him to. In chapter 3 I want to explore in more detail how such re-identifications work, and in particular discuss their relevance for an analysis of the notion of understanding 
referring acts. Does a hearer, in order to be credited with having understood a speaker's referring act, have to link the speaker's use of a referring expression to some idea which he already possesses? And if such a link has to be established by the hearer, does it have to be established in a certain way? What I will argue is that with regard to certain kinds of referring acts such link-ups are required. Yet even with regard to them this presents only a necessary condition for communicative success but not a sufficient one. The main requirement for communicative success seems to be that the hearer comes to entertain an idea which relates in some way to the speaker's underlying idea. In the remaining chapters I will try to spell out in more detail what this success-relation might consist in.

Intuitively, it is sameness in mental reference which accounts for the relevant success relation. ${ }^{5}$ That is, the hearer has to entertain an idea which is of the same object as is the speaker's underlying idea in order to be credited with having understood the speaker's referring act. Before the prospects of this proposed success relation can be discussed it will be necessary to make the conditions for mental reference of ideas or mental states more precise. Chapter 4 will be concerned with this topic. The central accounts of mental reference will be discussed, which are the familiar satisfactional, causal and hybrid accounts. Satisfactional accounts of mental reference assume that an idea or thought will be of that object in the world which in some way gets distinguished or picked out by it; causal accounts suppose that an idea will be of that object which has played a certain causal role in its production; hybrid accounts simply attempt to combine these elements in some way. It will be argued that pure satisfactional and causal accounts both fail, and that we need a hybrid account of mental reference. I will discuss Evans' (1982) hybrid account and Devitt and Sterelny's (1987) one - yet it will be argued that they both fail. What will be proposed as an alternative is a hybrid account according to which thinking of an object will be a matter of degrees.

On the basis of these considerations regarding the conditions of mental reference I will turn in chapter 5 to the question of understanding referring acts. What will be discussed first is the simple object-dependent account according to which a referring act succeeds if and only if speaker and hearer come to entertain ideas or thoughts which refer to the same object in the world. As has been stated above, such an account seems to be invalidated by cases like the camera-example, where communication seems to fail although the communicating agents' ideas refer to the same object, or the Troyexample, where communication seems to succeed although the

\footnotetext{
${ }^{5}$ The term 'mental reference' has been employed by Fitch (1990). Kvart (1994) uses the term 'thinker reference'.
} 
communicating agents' ideas refer to no object. It will be explored whether these and similar cases can be captured by employing a more "cosmopolitan" notion of reference, which allows for reference to fictional or unreal objects. For instance, one might think that communicative success in cases like the Troy-example can be accounted for by assuming that there has been shared mental reference to a fictional or unreal object. What I will argue is that the proposed defense fails as a general solution to the problems with a simple object-dependent account.

Then in the second part of chapter 5 a prominent alternative account of success in referential communication will be discussed, namely the one which can be seen as deriving from Frege's writings and which has been called Fregean account above. According to this account the hearer, in order to be credited with having understood the speaker's referring act, has to entertain the same mode of presentation as did the speaker when performing the referring act. What I will argue is that given certain widely held assumptions regarding how modes of presentation are to be individuated and ascribed, the Fregean account of success in referring acts turns out to be too strong. For in many cases where we intuitively think that the hearer has understood the speaker's referential use of a singular term, no understanding would occur since the associated modes of presentation are simply distinct. What might be suggested is to relax the Fregean communication-inducing relation that is supposed to hold between the entertained modes of presentation: instead of requiring them to be identical one might argue that they only have to be sufficiently similar. The prospects of this strategy of defense will be considered in more detail but it will be argued that it fails as well.

This justifies the search for an alternative account of understanding referring acts. In chapter 6 I will turn to a prominent one, namely to Evans' account which he proposed in his 1982 book 'The Varieties of Reference'. The account is a hybrid account in the sense that some sort of external success criteria get combined with more cognitive ones. That is, for a referring acts to succeed speaker and hearer must not only come to entertain thoughts that are externally related in the right way, but in addition they must also employ the right modes of presentation. Evans distinguishes between two kinds of referential communication, namely between cases of genuine referential communication and cases of quasi or make-believe referential communication. By the latter he means cases where agents use singular terms within the pretence of there being certain objects referred to while knowing that there are none. The two kinds of referential communication are assumed to have different external success conditions. For cases of genuine referential communication to succeed the hearer must 
come to entertain an idea or thought which refers to the same object as does the speaker's underlying idea or thought. On the other hand, for cases of quasi referential communication to succeed the hearer must for instance come to entertain a thought or idea which has the same causal origin or source as the speaker's underlying idea or thought. Although this account fares much better than any simple object-dependent or Fregean account in dealing with the empty cases like the Troy-example, I will argue that it nevertheless fails. The central problem is that there are many empty cases where on the one hand communication seems to succeed, yet which on the other hand do not qualify as cases of quasi referential communication.

In chapter 7 a new account of success in referential communication will be proposed which will circumvent the problems discussed in previous chapters. The account will resemble, in two important respects, Evans' one: first, it is a hybrid account in the sense that a sort of external success criterion gets combined with a cognitive one. Secondly, understanding referring acts will, in certain cases, also be considered as object-dependent. Yet the new account departs also from Evans' one in crucial ways. To start with, a different cognitive success condition will be put forward which will allow us to capture cases like the above camera-example. Secondly, the distinction between cases of referential communication which are objectdependent and those which are not will be drawn along different lines. As pointed out above, Evans distinguishes between genuine and quasi or let's pretend cases of referential communication. The former cases are considered by him to be object-dependent whereas the latter are not. I will argue that Evans is right with regard to the let's pretend cases but not with regard to remaining ones. They do not form a homogeneous group but different kinds can be distinguished. First there are the perception-based cases in which one intends to refer an audience to an object one is currently perceiving or one has perceived before. Such cases will be considered as object-dependent. Yet there are also the communication-based cases of referential communication, in which one intends to refer the audience to an object one has heard of from others. Such cases are not object-dependent. The central task of chapter 7 is to make this new account of understanding referring acts more precise. In the first part the focus will be on the proposed external success conditions for the different kinds of referential communication. Then in the second part I will turn to the Fregean side of the proposed hybrid account of understanding referring acts, namely to the question regarding how the associated modes of presentation must be related in order for a referring act to succeed. The proposals by Evans (1982) and Recanati (1993) will be discussed, yet it will be argued that they both fail. An alternative Cognitive success condition will be proposed which appeals to the idea of modes of 
presentations being compatible in an action initiating way. That is, the hearer in order to be credited with having understood the referring act by a speaker must come to entertain a mode of presentation which provides the basis for coordinating actions with the speaker.

Before jumping directly into the discussion of referring acts and of communicative success herein it will be important to lay down in more detail the methodology of the task. Which standards or criteria does an account of success in referential communication have to fulfil in order to be counted as adequate?

\section{THE METHODOLOGY}

Intuitively it is plausible that an account of understanding referring acts should accord with our intuitions, namely with those regarding agents understanding or failing to understand such communicative acts. In fact, in criticizing certain accounts of understanding referring acts above I have already appealed quite extensively to intuitions of this kind. For instance it has been pointed out that an object-dependent account of success in referring acts which makes communicative success dependent on agents' thoughts referring to the same object in the world obviously fails because it clashes with the intuition that there can be communicative success in cases where nothing gets referred to. In fact, this and some other intuitions have been presented as central constraints which any account of success in referential communication has to accord with in some way. Yet one might wonder why these intuitions have been taken so seriously? Why can an account of understanding referring acts be ruled out simply because it stands in conflict with certain intuitions of ours? In most other sciences for example no one would give up a theory simply because it clashes with certain common sense intuitions. Thus the question is: what is so important about our intuitions in accounting for success in communication?

According to a dominant philosophical tradition which has taken conceptual analysis as the proper business of philosophy these intuitions simply present the data or facts one's analysis has to accord with. ${ }^{6}$ For, so the reasoning will go, what one is after here is the elucidation of a certain concept, namely the concept of understanding referential uses of singular terms in communication. But the conditions for the correct application of this concept, as in other cases of conceptual analysis, can only be revealed on the basis of our intuitions regarding its applications, i.e. on the basis of

\footnotetext{
${ }^{6}$ See Strawson (1992) for a nice statement of this widely accepted methodology, in particular his chapter 1.
} 
our intuitions regarding success and failure in referential communication. And thus these intuitions figure as data.

But one might wonder whether the question regarding the conditions under which referring acts are successful really expresses a quest for conceptual analysis? For is it not the case that what one is after here is the development of a kind of scientific theory of communication? And in this sense the aim is not to reveal our concept of agents understanding referring acts but to say something about the world, namely about the phenomenon of successful communication which takes place in it as other phenomena like earthquakes, lunar eclipses, the spread of viruses and racial discriminations do. As Devitt (1985, 1994), Dennett (1987) and others have argued, what one is trying to do in philosophy is frequently a form of protoscience, that is the attempt to lay the foundations of a new discipline. In our case this would be the discipline of communication studies. Accordingly our intuitions should have the same status here as they have in the beginning age of any other discipline, which Devitt (1994) describes as follows:

"They are part of an empirical, fallible, and certainly inadequate set of folk opinions, or more pretentiously, 'folk theory', the linguistic wisdom of the ages." (p. 547)

But still as such our intuitions are, as Devitt (1985) says, the "suitable starting point" in the development of a new discipline. Thus they present also some sort of data. Yet in contrast to the role they play within the conceptual analysis paradigm they are not sacrosanct since they might be invalidated in the light of other considerations.

Although I tend to agree more with Dennett's and Devitt's approach towards the role of intuitions in accounting for the success in referring acts it does not matter very much where one stands on this issue. For also under a more scientifically orientated approach our intuitions regarding agents succeeding in communication will play a central role as data. The only difference to the conceptual analysis paradigm is that they are not sacrosanct. But even this assumption seems to be given up by philosophers doing conceptual analysis, namely when they distinguish between tutored and untutored intuitions and treat only the former ones as decisive for their analyses. The untutored ones are the raw material one is confronted with when ascertaining agents' intuitive judgements regarding the application of a concept. But in the light of some theoretical guidance agents might revise these judgements and come up with different ones, the tutored ones. Thus I think the following descriptive adequacy criterion will be in agreement with the two general stances regarding the claim "intuitions are data": 
Descriptive Adequacy Criterion: An account of communicative success in referring acts has to accord with our intuitions of agents understanding and failing to understand such acts as long as these intuitions are in agreement with other well-motivated considerations.

This criterion stresses the importance of our intuitions in judging the adequacy of any account of what it is to succeed in referring acts. Yet it also acknowledges that these intuitions might in certain cases be invalidated in the light of some other considerations. It should be noted that these considerations must be well-motivated, i.e. whenever one intends to ignore certain intuitions or types of intuitions motivation has to be given. This is to rule out attempts to ignore certain intuitions just because they harm one's analysis. In case where no further reasons can be given for ignoring them such an analysis is simply inadequate. Further it is important to point out that these "referential intuitions" must not necessarily support a one-folded account of success in referential communication according to which there is only one notion of communicative success. For instance they might point to a two-folded notion or even to a graded notion of communicative success, i.e. there would not be simply communicative success but communicative success in a certain respect or to a certain degree. Later we will see that this indeed presents a plausible view on communication which will allow us to circumvent certain problems.

To sum up these methodological remarks, for an account of communicative success in referring acts to be adequate it has to capture our intuitions of agents succeeding or failing in these acts, at least to a certain degree. Besides general constraints on any satisfactory analysis or account of some phenomena, for instance that it is explanatory, these intuition will play throughout this work the central role in evaluating the adequacy of accounts of referential communication. After having made these methodological remarks let me point out briefly the empirical and computational relevance of an inquiry into the question of what communicative success in referring acts consists in.

\section{THE EMPIRICAL RELEVANCE}

In studying communication philosophers have usually discussed the question of what communicative success consists in, let us call it the what-question, whereas empirical and computational research has focused predominantly on the question regarding how agents achieve communicative success, or simply the how-question. Three influential approaches regarding this latter question can be distinguished. First, there is the traditional code model 
according to which agents communicate simply by encoding and decoding signals like language expressions. This model, which some date back to Aristotle, has found its modern expression in the work of Shannon \& Weaver (1949) and in the semiotic tradition; see Saussure (1959) or Eco (1984). Yet many like Grice $(1957,1975)$ or later Sperber \& Wilson (1986) have argued that achieving success in communication involves more than simply the decoding of certain signals. In addition inferential elements play a central role. This second model of communication which stresses these inferential elements has been called, not surprisingly, the inferential model. In recent years yet a third model has emerged which puts emphasis on another element in communication, namely collaboration. It takes notice of the fact that in communication agents do not, in many cases, achieve communicative success simply by working on their own, but only by collaborating in one form or other with each other, for instance by asking questions, making corrections in the light of reactions of their communication partners, etc. A model which tries to incorporate these elements can be called the collaborative model and has been developed by psycholinguists like Clark (1992) or Anderson et al. (1991).

It seems plausible that there is some truth in each of these models of how agents achieve communicative success. For agents obviously make use, to a certain extent, of codes, inferences and collaboration in achieving communicative success. Yet the question is how these elements are related: when do agents make use of collaboration, and what are the details with regard to these elements? For example, what sorts of collaborative strategies do agents exploit in achieving communicative success? In the last two decades psycholinguists and artificial intelligence researchers have set out to answer these questions systematically. In empirical dialogue studies for instance, the strategies agents pursue in achieving communicative success and in particular the extent to which they collaborate herein have been explored in more detail; see Anderson et al. (1991) or Clark (1992) for instance. Artificial Intelligence researchers like Hirst (1993) or authors in Cohen et al. (1990) have tried to use those results or to come up with their own in developing artificial communication devices that can engage in successful human-machine communication. Yet no full-blown theory like a "mechanics of communication" which gives a detailed account of how successful communication is achieved has been developed so far. Certainly one reason for this situation is the infancy of the field. Yet another reason might be that no satisfying answer to the question of what communicative success consists in has been given so far. That such an answer is highly relevant for the empirical as well as the computational research can be shown as follows: 
First, in order to do experiments into how agents communicate successfully one needs operational criteria in order to decide when success has been achieved. The central question with regard to a proposed set of operational criteria will always be whether they in fact measure success in communication. There are two ways in which a set of operational criteria can be adequate: (a) they are consistent in the sense that whenever the criteria measure communicative success there is success or (b) they are complete in the sense that whenever there is communicative success it can be measured by the criteria. Now it is obvious that in evaluating consistency as well as completeness of a set of operational criteria one must rely on one's conception of communicative success. For how would one decide that in some case there is communicative success if not on the basis of one's conception of what communicative success is? Secondly, within the research paradigm of Artificial Intelligence the central question is the following: which representation base and which algorithm will enable a computer system to achieve communicative success? Two strategies have been proposed in answering this question: (a) answering it by copying successful human communication strategies, and (b) answering it by treating it like any ordinary computational problem. Yet it is obvious that both strategies also presuppose a conception of what successful communication is, that is an answer to the what-question. With regard to (a) such a conception is needed because in order to find out about the human strategies one has to rely on empirical research which in turn presupposes an account of the whatquestion, as has been argued before. With regard to (b) an answer to the what-question is needed since the possibilities of a computational solution can only be explored on the basis of an explicit characterization of what one is aiming at, which just is success in communication. Thus to conclude, it seems that there will not be a justified and complete answer to the howquestion without an answer to the question regarding what communicative success consists in. In this work the focus will almost be exclusively on this latter question, yet the hope is that in accounting for it a contribution to the empirical and computational work might be made as well. 